\title{
PHENOTYPIC DETECTION OF EXTENDED-SPECTRUM BETA LACTAMASES (ESBLS) PRODUCING ORGANISMS AMONG ENTEROBACTERIACEAE AND THEIR ANTIBIOTIC RESISTANCE PATTERN IN URINARY TRACT INFECTION (UTI) AT KUMAUN REGION, UTTARAKHAND
}

\author{
Hitendra Singh ${ }^{1}$ Umesh $^{2}$
}

${ }^{1}$ Assistant Professor, Department of Microbiology, Government Medical College, Haldwani, Uttarakhand.

2Professor, Department of Microbiology, Government Medical College, Haldwani, Uttarakhand.

\begin{abstract}
BACKGROUND
ABSTRACT

Urinary tract infection (UTI) is one of the major health problems affecting all age groups. Extended Spectrum $\beta$-Lactamases (ESBLs) producing Enterobacteriaceae exhibits a resistant mechanism, which challenges the strategies of broad-spectrum antibiotics used in treatment of UTI, thus limiting therapeutic options. Knowing resistance pattern of such multidrug resistant pathogens will help in the appropriate usage of antimicrobial agents.

The study was carried out to determine the prevalence of ESBL among the Enterobacteriaceae uropathogens and their antimicrobial resistance pattern.
\end{abstract}

\section{MATERIALS AND METHODS}

All the uropathogenic isolates obtained from symptomatic UTI cases were identified by conventional methods. The prevalence of potential ESBL producers among Enterobacteriaceae isolates was explored. Antimicrobial susceptibility testing was done by KirbyBauer disc diffusion method and the results were interpreted according to Clinical Laboratory Standards Institute 2014 guidelines. Study Design- Descriptive study.

Place and Duration of Study- Department of Microbiology, Government Medical College and Hospital, Haldwani from November 2013 to September 2015.

\section{RESULTS}

A total of 695 clinical isolates were obtained from 2438 urine samples. Out of these 695 isolates 454 (65.32\%) isolates were from Enterobacteriaceae family, out of which a total of $188(41.4 \%)$ isolates were ESBLs producing Enterobacteriaceae which predominantly comprised of Klebsiella pneumoniae (65.71\%) followed by Escherichia coli (40.56\%) and Proteus species (34.28\%). A high degree of resistance to ampicillin (98.4\%) and cotrimoxazole (93.08\%) were found among these ESBL producing Enterobacteriaceae isolates followed by gentamicin (58.51\%) and ciprofloxacin (55.31\%). Nitrofurantoin and Amikacin were found to be most effective drugs.

\section{CONCLUSION}

A large number of uropathogens were found to be ESBL producers in the present study. Most of the ESBL producing isolates were multidrug resistant. Monitoring of ESBL production, judicious use of antibiotics and infection control measures are necessary to avoid treatment failure in patients with UTI.

\section{KEYWORDS}

Prevalence, Urinary Tract Infections, Extended Spectrum $\beta$-Lactamases, Enterobacteriaceae, Klebsiella Pneumoniae, Escherichia Coli.

HOW TO CITE THIS ARTICLE: Singh H, Umesh. Phenotypic detection of extended-spectrum beta lactamases (ESBLS) producing organisms among enterobacteriaceae and their antibiotic resistance pattern in urinary tract infection (UTI) at Kumaun region, Uttarakhand. J. Evolution Med. Dent. Sci. 2018;7(16):2014-2017, DOI: 10.14260/jemds/2018/453

\section{BACKGROUND}

Extended-spectrum $\beta$-lactamase (ESBLs) enzymes are plasmid-mediated enzymes with capacity to hydrolyse and thus inactivate broad-spectrum $\beta$-lactam antibiotics, which in turn confer a decreased susceptibility against commonly used antibiotic drugs, such as penicillins and extended-spectrum cephalosporins. ${ }^{[1]}$

'Financial or Other Competing Interest': None.

Submission 08-03-2018, Peer Review 01-04-2018,

Acceptance 07-04-2018, Published 16-04-2018.

Corresponding Author:

Dr. Hitendra Singh,

Flat No. 301, Type 3,

Government Doon Medical College Campus,

Dehrakhas, Patel Nagar,

Dehradun-248001, Uttarakhand.

E-mail: hitenhims@gmail.com

DOI: $10.14260 /$ jemds $/ 2018 / 453$
A wide range of Enterobacteriaceae family members express Extended-spectrum $\beta$-lactamase (ESBLs) enzyme. The prevalence of extended spectrum beta-lactamase (ESBL) producing organisms among clinical isolates vary greatly worldwide and is rapidly changing over time. Urinary tract infection is a common bacterial disease, often contributes to a frequent cause of morbidity in outpatients as well as hospitalised patients. ${ }^{[2]}$ Although, UTIs occur in both men and women, clinical studies suggest that the overall prevalence of UTI is higher in women. The introduction of antimicrobial therapy has contributed significantly to the management of UTIs. Still the major problem with routine antibiotic therapies is the rapid emergence of antimicrobial resistance in clinical settings and the community. ${ }^{[3]}$ The resistance pattern of community acquired uropathogens has not been extensively studied in the Indian subcontinent.[4-6] Detection of ESBL producing organism from samples such as urine may be important, because this represents an epidemiologic marker 
of colonisation and therefore there is potential for transfer of such organisms to other patients.[7] The study was carried out to determine the prevalence of ESBL among the Enterobacteriaceae uropathogens and knowing resistance pattern of such multidrug resistant pathogens will help in the appropriate usage of antimicrobial agents and prevent further emergence of resistant strains.

\section{MATERIALS AND METHODS}

A Descriptive study of total 2438 urine samples were processed from symptomatic UTI cases attending or admitted to the Government Medical College and Hospital, Haldwani from November 2013 to September 2015. Urine samples were inoculated onto cystine lactose electrolyte-deficient agar (CLED agar) using a fixed volume loop $(0.01 \mathrm{~mL}$ ). Culture plates were aerobically incubated at $37^{\circ} \mathrm{C}$ for 18 to 24 hrs. and examined for growth of pathogenic microorganisms. Colony count of $\geq 10^{5} \mathrm{CFU} / \mathrm{mL}$ was considered to be significant. The antibiotic discs (concentrations in $\mu \mathrm{g}$ ) included in the panel for Enterobacteriaceae isolates were ampicillin $(10 \mu \mathrm{g})$, gentamicin $(10 \mu \mathrm{g})$, amikacin $(30 \mu \mathrm{g})$, ciprofloxacin $(5 \mu \mathrm{g})$, levofloxacin $(5 \mu \mathrm{g})$, norfloxacin $(10 \mu \mathrm{g})$, nitrofurantoin $(300 \mu \mathrm{g})$ and cotrimoxazole $(1.25 / 23.75 \mu \mathrm{g})$. Ceftazidime $(30 \mu \mathrm{g})$ and ceftazidime/clavulanic acid (30 $\mu \mathrm{g} / 10 \mu \mathrm{g}$ ) were included with antibiotics panel to screening as well as for detection of ESBL producing Enterobacteriaceae. E-test strip was applied for confirmation of ESBL producing Enterobacteriaceae isolates. The discs were provided by Hi-Media Laboratories Pvt. Ltd., Mumbai.

\section{Tests for ESBL Production in Members of Family Enterobacteriaceae}

Isolates of family Enterobacteriaceae that were considered to be positive for ESBL production by the phenotypic confirmatory disc diffusion test (PCDDT) were subjected to the E-test-

1. CLSI Phenotypic Confirmation Test[8]- The ceftazidime $(30 \mu \mathrm{g})$ discs alone and in combination with clavulanic acid (ceftazidime + clavulanic acid, 30/10 $\mu$ g disc) were applied onto a plate of Mueller-Hinton Agar (MHA), which was inoculated with the lawn culture of the test strain. The plates were incubated overnight at $37^{\circ} \mathrm{C}$. An increase of $\geq 5 \mathrm{~mm}$ in the zone of inhibition of the combination disc in comparison to the ceftazidime disc alone was considered to be a marker for ESBL production.

2. E-test- Minimum inhibitory concentration (MIC) was determined for the ESBL Enterobacteriaceae isolates by E-test using ESBL strips (HiMedia Laboratories Pvt. Ltd., Mumbai). The ESBL E-strip is a plastic drug-impregnated strip, strips were impregnated with cefotaxime (CTX) at one end and cefotaxime + clavulanic acid (CTX+) at another end. A lawn culture of the test organism was inoculated on Mueller-Hinton Agar (MHA). The E-test ESBL strip was placed on the centre of the plate. The plates were incubated aerobically at $37^{\circ} \mathrm{C}$ for $16-18$ hours. The MIC was interpreted as the value at the intersection of the growth ellipse with the strip. The isolate was confirmed to be an ESBL producer when the ratio of the MIC value of cefotaxime to the MIC value of cefotaxime in combination with clavulanic acid was more than 8. The ESBL production was also confirmed when no zone was obtained for cefotaxime, but zone was observed in cefotaxime and clavulanic acid combination.

\section{Control}

Escherichia coli ATCC 25922 were used as the negative control and Klebsiella pneumoniae ATCC 700603 was used as the positive control.

\section{Date Entry and Analysis}

Data entry will be done in MS Excel and Statistical analysis was done. Among the Enterobacteriaceae isolates obtained from sample, screening for ESBL producing Enterobacteriaceae was done and quantified in percentages and tabulated. Antibiotic resistance pattern of ESBL producing Enterobacteriaceae isolates obtained was quantified in percentage and tabulated.

\section{RESULTS}

A total of 695 clinical isolates were obtained from 2438 urine samples. Out of these 695 isolates 454 (65.32\%) isolates were from Enterobacteriaceae family, out of which a total of 188 (41.4\%) isolates were ESBLs producing Enterobacteriaceae.

Among the ESBL producing Enterobacteriaceae, Escherichia coli was found in maximum number of isolates. Whereas the higher prevalence detected in Klebsiella pneumoniae (65.71\%) followed by Escherichia coli (40.56\%), Klebsiella oxytoca (37.5\%), Proteus species (34.28\%), Citrobacter freundii (29.41\%) and Providencia rettgeri (25\%). A high degree of resistance to ampicillin and cotrimoxazole were found among these ESBL producing Enterobacteriaceae isolates $\quad(98.4 \%$ and $93.08 \%$ respectively). High degree of resistance was also seen with fluoroquinolones and gentamicin. The most susceptible drug against these strains was nitrofurantoin and amikacin, which showed $15.95 \%$ and $25 \%$ resistances respectively.

\begin{tabular}{|c|c|c|c|}
\hline 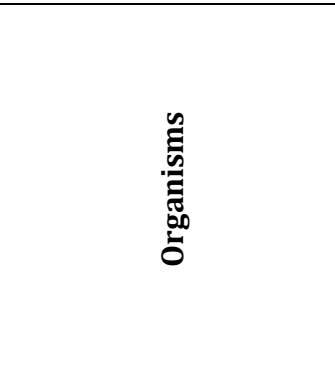 & 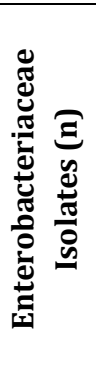 & 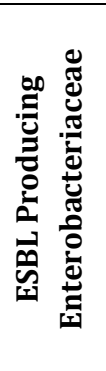 & 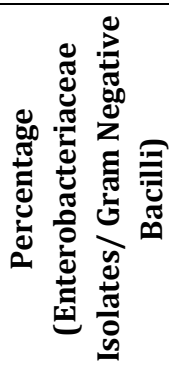 \\
\hline E. coli & 355 & 144 & $40.56 \%$ \\
\hline Klebsiella pneumoniae & 35 & 23 & $65.71 \%$ \\
\hline Klebsiella oxytoca & 8 & 3 & $37.5 \%$ \\
\hline Proteus mirabilis & 23 & 10 & $43.47 \%$ \\
\hline Proteus vulgaris & 12 & 2 & $16.66 \%$ \\
\hline Citrobacter freundii & 17 & 5 & $29.41 \%$ \\
\hline Providencia rettgeri & 4 & 1 & $25.0 \%$ \\
\hline Total & 454 & 188 & $41.4 \%$ \\
\hline \multicolumn{4}{|c|}{$\begin{array}{c}\text { Table 1. Screening of ESBL producing Enterobacteriaceae } \\
\text { Isolates }\end{array}$} \\
\hline
\end{tabular}




\begin{tabular}{|c|c|c|}
\hline Antibiotics & $\begin{array}{c}\text { Resistance } \\
\text { Isolates (n) }\end{array}$ & $\begin{array}{c}\text { Percentage } \\
\text { (\%) }\end{array}$ \\
\hline Ampicillin & 185 & $98.4 \%$ \\
\hline Gentamicin & 110 & $58.51 \%$ \\
\hline Amikacin & 47 & $25 \%$ \\
\hline Levofloxacin & 77 & $40.95 \%$ \\
\hline Ciprofloxacin & 104 & $55.31 \%$ \\
\hline Norfloxacin & 130 & $69.14 \%$ \\
\hline Nitrofurantoin & 30 & $15.95 \%$ \\
\hline Cotrimoxazole & 175 & $93.08 \%$ \\
\hline $\begin{array}{c}\text { Table 2. Antibiotic Resistance Pattern of ESBL producing } \\
\text { Enterobacteriaceae Isolates }\end{array}$ \\
\hline
\end{tabular}

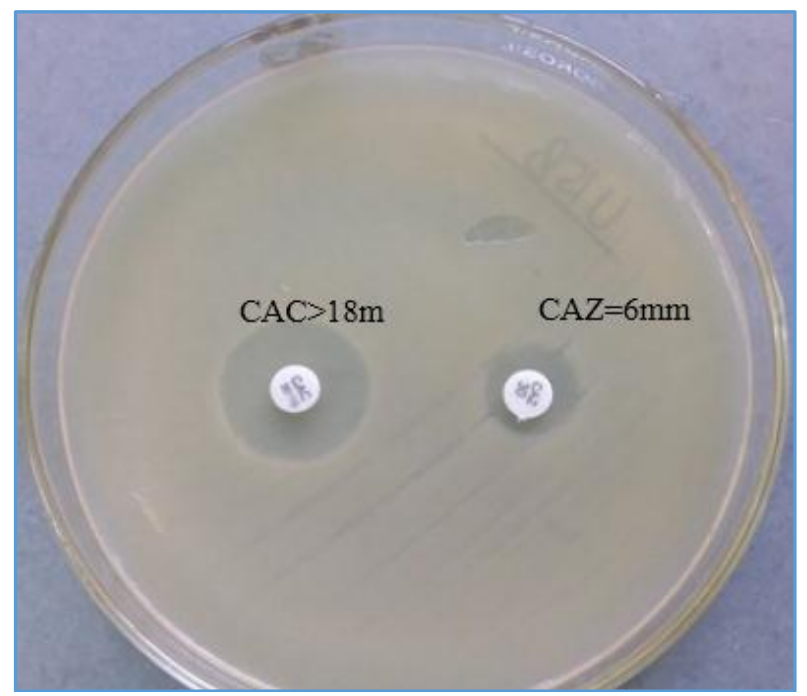

Figure 1. A Photograph showing Production of ESBL by Phenotypic Confirmatory Disc Diffusion Test, Ceftazidime Clavulanic Acid (CAC) and Ceftazidime (CAZ)

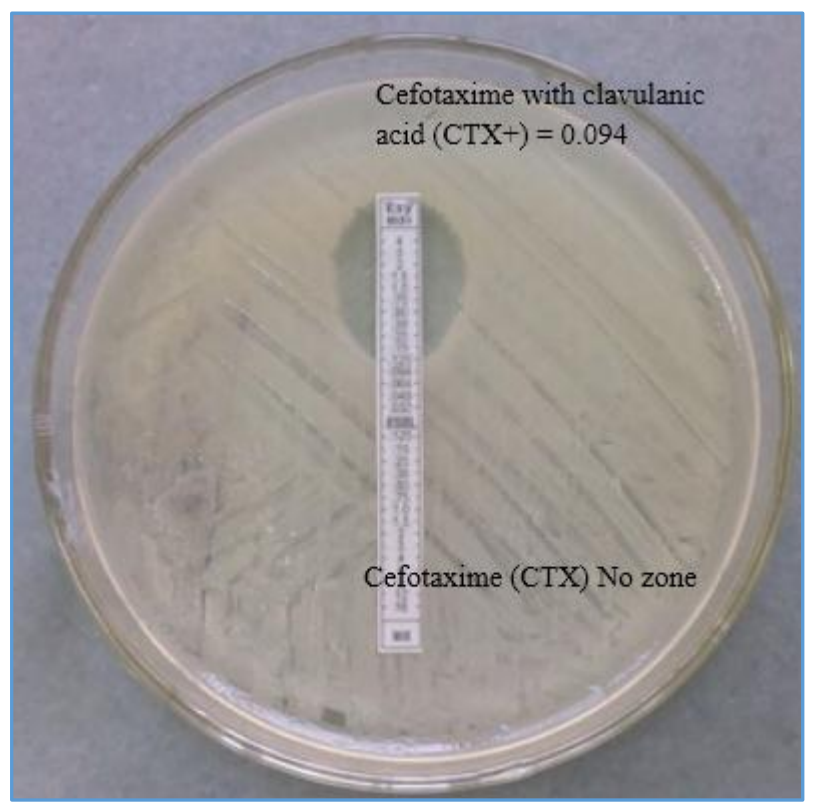

Figure 2. A Photograph showing Production of ESBL by E-Test

\section{DISCUSSION}

In the current era, extended-spectrum $\beta$-lactamases (ESBLs) organisms are a major global problem in the clinical and community settings due to the increasing use of broadspectrum antimicrobial agents. The total prevalence of ESBL producing Enterobacteriaceae was found to be $41.4 \%$ in our study, which was approximately equal to Tillekeratne et al[9] and Metri et al,[10] which reported the ESBL prevalence of $40.2 \%$ and $39.1 \%$ respectively in urine specimen among Enterobacteriaceae. Hanan A et al[11] and Shashwati et al[12] also reported $51 \%$ and $50 \%$ prevalence respectively. Mohanty et al[13] reports the higher prevalence of $68.78 \%$ for ESBL producing Enterobacteriaceae organisms. This study showed the degree of ESBL prevalence is high among Klebsiella pneumoniae $(65.71 \%)$ followed by E. coli (40.56\%), Proteus spp. (34.28\%), Citrobacter freundii (29.41\%) and Providencia spp. (25\%). Afridi F et al[14] study showed highest frequency for ESBL production in Klebsiella species (84.61\%) followed by Escherichia coli (68.55\%), Enterobacter species (36.84\%) and Proteus mirabilis (28.57\%). Ahmed et al[15] reported frequency rates among Klebsiella species (40\%) followed by Escherichia coli (30\%), Proteus spp. (16\%) and Enterobacter species (14\%). Khurana et al[16] reported Klebsiella species prevalence of 38.5\% followed by $24.7 \%$ of Escherichia coli in urinary isolates from hospitalised patients, while Mathur et al[17] reported $80 \%$ prevalence of Klebsiella species as a most frequent ESBL producing organism. In a study concluded by Shobha et al,[18] she states that Citrobacter spp. was the third most common urinary pathogen and $30 \%$ of the isolates were extended spectrum beta lactamase (ESBL) producers. The prevalence of ESBL producers was found to vary greatly in different areas of India. ESBL producing strains often arise in focal outbreaks. Regional and local estimates are probably more useful than are more global assessments in clinical decision making and for infection control purpose.[14] All isolates were applied for antibiotics sensitivity testing and nitrofurantoin was found to be the most effective drug. It showed $15.95 \%$ resistance to all isolates, whereas Amikacin was the second most effective drug which showed $25 \%$ resistance. Resistance to ampicillin was $98.4 \%$ followed by cotrimoxazole (93\%), norfloxacin (69.14\%), gentamicin (58.51\%), ciprofloxacin (55.31\%) and levofloxacin (40.95\%). Garcia A et al[19] reported nitrofurantoin was a most effective drug, it showed $10.9 \%$ resistance. This study also reported $62 \%, 84.8 \%$ and $37 \%$ resistance to cotrimoxazole, ciprofloxacin and gentamicin respectively. Shashwati $\mathrm{N}$ et al[12] showed $50 \%$ resistance against gentamicin, $87.5 \%$ to ciprofloxacin and $94.65 \%$ to trimethoprim/ sulfamethoxazole. This study suggests that the use of these antibiotics for common illness should be avoided and the drug should be reserved as a second line drug. Nitrofurantoin, which is excreted primarily in urine and used also to treat UTI in pregnancy had also been found as the most effective drug against these isolates. Among other antibiotics, resistant isolates against norfloxacin and ciprofloxacin were found to be more than the aminoglycosides antibiotics. Therefore, aminoglycosides antibiotics can be used to treat the urinary infections on the empirical basis. ESBL producing Enterobacteriaceae are on rise and there are various factors that attribute to such kind of resistance. In this study, ESBL producing Enterobacteriaceae were studied in urinary samples and factors that can lead to rise in this type of resistance in urinary tract infections include old age, prolong bladder catheterisation, recurrent infection, long hospital stay, irrational use of antimicrobial agents, non-compliance of patient and presence of comorbidities. 


\section{CONCLUSION}

Multidrug resistance was significantly higher in ESBL positive isolates. Knowledge of the prevalence of ESBLs and resistance pattern of bacterial isolates in a geographical area is of utmost importance. The sensitivity pattern of microorganisms to various antibiotics varies over time and among different geographical locations. Therefore, continuous analysis of the antibiotic resistance pattern acts as a guide in initiating the empirical treatment of UTI and the therapy must be started. Only urine culture and sensitivity have been done. It helps in avoiding the treatment failure.

\section{REFERENCES}

[1] Bradford PA. Extended-spectrum beta-lactamases in the 21st century: characterization, epidemiology, and detection of this important resistance threat. Clin Microbiol Rev 2001;14(4):933-51.

[2] Wagenlehner FM, Naber KG. Treatment of bacterial urinary tract infections: presence and future. Eur Urol 2006;49(2):235-44.

[3] Habte TM, Dube S, Ismail N, et al. Hospital and community isolates of uropathogens at a tertiary hospital in South Africa. S Afr Med J 2009;99(8):584-7.

[4] Akram M, Shahid M, Khan AU. Etiology and antibiotic resistance patterns of community-acquired urinary tract infections in JNMC Hospital, Aligarh, India. Ann Clin Microbiol Antimicrob 2007;6:4.

[5] Kothari A, Sagar V. Antibiotic resistance in pathogens causing-community acquired urinary tract infections in India: a multicentric study. J Infect Dev Ctries 2008;2(5):354-8.

[6] Biswas D, Gupta P, Prasad R, et al. Choice of antibiotic for empirical therapy of acute cystitis in setting of high antimicrobial resistance. Indian J Med Sci 2006;60(2):53-8.

[7] Willinger B, Manafi M. Evaluation of a new chromogenic agar medium for the identification of urinary tract pathogens. Lett Appl Microbiol 1995;20(5):300-2.

[8] Clinical and Laboratory Standards Institute. Performance standards for antimicrobial susceptibility testing. Twenty four informational supplement ed. CLSI document M100-S24. Wayne, PA: CLSI 2014.

[9] Tillekeratne LG, Vidanagama D, Tippalagama R, et al. Extended-spectrum ß-lactamase-producing entero bacteriaceae as a common cause of urinary tract infections in Sri Lanka. Infect Chemother 2016;48(3):160-5.
[10] Metri C, Peerapur JP, Basavaraj V. The prevalence of ESBL among enterobacteriaceae in a tertiary care hospital of north Karnataka, India. Journal of Clinical and Diagnostic Research 2011;5(3):470-5.

[11] Hanan A. Detection of extended-spectrum blactamases in members of the family enterobacteriaceae at a teaching hospital, Riyadh, Kingdom of Saudi Arabia. Saudi Med J 2002;23(2):18690.

[12] Shashwati N, Kiran T, Dhanvijay AG. Study of extended spectrum $\beta$-lactamase producing enterobacteriaceae and antibiotic coresistance in a tertiary care teaching hospital. J Nat Sci Biol Med 2014;5(1):30-5.

[13] Mohanty S, Singhal R, Sood S, et al. Comparative in vitro activity of beta-lactam/beta-lactamase inhibitor combinations against gram negative bacteria. Indian J Med Res 2005;122(5):425-8.

[14] Afridi FI, Farooqi BJ, Hussain A. Frequency of extended spectrum beta lactamase producing enterobacteriaceae among urinary pathogen isolates. J Coll Physicians Surg Pak 2011;21(12):741-4.

[15] Ahmed E, Bugti S, Shaikh AA, et al. Isolation and drug sensitivity of extended spectrum beta lactamase (ESBL) uropathogens. Journal of Rawalpindi Medical College 2015;19(2):155-8.

[16] Khurana S, Taneja N, Sharma M. Extended spectrum beta-lactamase mediated resistance in urinary tract isolates of family enterobacteriaceae. Indian J Med Res 2002;116:145-9.

[17] Mathur P, Kapil A, Das B, et al. Prevalence of extended spectrum beta lactamase producing gram negative bacteria in a tertiary care hospital. Indian J Med Res 2002;115:153-7.

[18] Shobha KL, Gowrish Rao S, Rao S, et al. Prevalence of extended spectrum Beta Lactamases in urinary isolates of Escherichia coli, klebsiella and citrobacter species and their antimicrobial susceptibility pattern in a tertiary care hospital. Indian J Practising Doctor. 3 2007-01- 2007-02.

[19] García-Tello A, Gimbernat H, Redondo C, et al. Extended-spectrum beta-lactamases in urinary tract infections caused by enterobacteria: understanding and guidelines for action. Actas Urol Esp 2014;38(10):678-84. 